\title{
Impacto de las distintas estrategias de tratamiento de observación directa y supervisada para tuberculosis
}

Impact of DOTS compared with DOTS-plus on multidrug resistant tuberculosis and tuberculosis deaths:decisión analysis. Sterling T, Lehmann H, Frieden T. BMJ. 2003; 326:574 (15 March)

\section{Objetivo}

Evaluar en el mundo subdesarrollado el impacto en la muerte por tuberculosis de la estrategia de tratamiento de observación directa y supervisada (TODS) comparándola con la TODS plus.Ver cuadro con definiciones.

Cuadro 1: definiciones de las distintas formas de terapia de observación directa y supervisada para el tratamiento de la tuberculosis.

- La terapia de observación directa y supervisada (TODS) implica el compromiso gubernamental para la pesquisa con examen directo de esputo de los pacientes con sospecha de tuberculosis en los mismo servicios de atención primaria; el tratamiento cortos (seis meses) con drogas de primera línea (isoniazida, rifampicina, pirazinamida y etambutol o estreptomicina) de los casos positivos; la búsqueda de nuevos casos entre los contactos; el abastecimiento adecuado de las drogas; el monitoreo de los planes; etc. En los países subdesarrollados no suelen hacerse cultivos ni antibiogramas y, por lo tanto, no suele documentarse la emergencia de cepas resistentes. Es rara la utilización de drogas de segunda línea.

- La terapia de observación directa y supervisada (TODS) plus agrega el uso de tratamientos de segunda línea (más tóxicos y caros) por 18 a 24 meses utilizando por lo menos dos drogas a las que se haya documentado susceptibilidad incluyendo una en forma parenteral. La decisión del uso de drogas de segunda línea puede ser en base al antibiograma o al fracaso del tratamiento con drogas de primera línea.

\section{Diseño}

Análisis de decisión tipo Markov* con simulación de Monte Carlo.

\section{Lugar}

Centro Universitario de Investigación en Tuberculosis de John Hopkins, Baltimore, Estados Unidos.

\section{Medición de resultados principales}

El número acumulado de muertes por tuberculosis por 100,000 habitantes en 10 años.

\section{Resultados principales}

En un área con una prevalencia de $3 \%$ de tuberculosis multidrogoresistente primaria y bajo la estrategia de TODS implementada en óptimas condiciones, 276 personas morirán de tuberculosis en 10 años (24 multidrogo resistentes y 252 no multidrogo resistentes). La implementación óptima de la TODS plus evitaría cuatro de estas muertes.

Si la implementación de TODS plus diera por resultado una disminución de solo el $5 \%$ de la efectividad de la estrategia TODS, moriría un $16 \%$ más gente con tuberculosis.

\section{Conclusiones}

En óptimas condiciones de implementación, con la estrategia TODS plus pueden evitarse muertes por tuberculosis si se la compara con la estrategia TODS.Sin embargo, si las dificultades de implementación de la TODS plus estuvieran asociadas con una mínima reducción en la efectividad del tratamiento, morirían sustantivamente más personas que bajo la TODS.

Fuente de financiamiento:National Institute of Allergy and Infectous Disesases.

\section{Comentario}

Este análisis de decisión muestra la importancia de las estrategias de tratamiento directamente observado en tuberculosis (estrategias TODS y TODS plus) en la disminución de la muertes por esta enfermedad. Destacamos la necesidad de que los programas de tuberculosis implementen TODS efectivos antes implementar TODS plus, no solamente por el mayor costo que implican estos últimos sino por los recursos humanos y logísticos que requieren. Si bien llama la atención que no se haya considerado el beneficio que implica prevenir la transmisión de multidrogo resistentes con el tratamiento efectivo a través del TODS plus, es también cierto que un efectivo TODS también puede disminuir el riesgo de aparición de multidrogo resistentes.

Quedan algunas dudas ya que un análisis de decisión bajo el modelo Markov no puede representar, por ejemplo, el grado de transmisión de enfermedad, especialmente en países donde la prevalencia e in- cidencia de tuberculosis es elevada o en regiones del mundo donde el porcentaje de multidrogo resistente son muy altos.

Los resultados de este análisis de decisión son una alerta para aquellos programas de tuberculosis que en la actualidad usan ambas estrategias ya que la TODS plus restaría recursos a la TODS, lo que podría conducir a que termine habiendo más muertes por tuberculosis y más individuos enfermos con cepas altamente resistentes a las drogas.

Conclusiones del comentador: Concluimos que los estados deben esforzarse en implementar ambas estrategias efectivamente y con los recursos necesarios ya que las estrategias de tratamiento directamente observado han demostrado resultados importantes ${ }^{1,2,3,4,5}$ en la curación y disminución de la mortalidad por tuberculosis.

\section{Dr. Juan Spelucin [ Dirección Regional de Salud - Cusco - Perú ]}

Referencias:

1.Telzak EE, Sepkowitz K, Alpert P, Mannheimer S, Medard F, El-Sadr W, et al.Multidrug-resistant tuberculosis in patients without HIV infection.N Engl J Med 1995;333:907-911 2. Espinal MA, Kim SJ, Suarez PG, Kam KM, Khomenko AG, Migliori GB, et al.Standard short-course chemotherapy for drug-resistant tuberculosis.Treatment outcomes in 6 countries.JAMA 2000;283:2537-2545

3.Mitnick C, Bayona J, Palacios E et al.Community-based therapy for multidrug-resistant tuberculosis in Lima, Peru.N Engl J Med 2003;348:119-28.

4.Tahaoglu K, Torun T, Sevin T, et.al.The treatment of multidrug- resistant tuberculosis in Turkey.N Engl J Med 2001;345:170-4.

5.Park SK, Kim CT, Song SD, et.al.Outcomes of chemotherapy in 107 patients with pulmonary tuberculosis resistant to isoniazid and rifampin.Int J Tuberc Lung Dis 1998;2:877-84. 